Pub. Mat. UAB

$N^{\circ} 21$ Oct. 1980

Actes VII JMHL

\title{
EXTENSIONES COFIBRACION-FIBRACION
}

\section{L.J. Hernández Paricio}

Dpto. de Geometría y Topologia

Universiad de Zaragoza

Llamamos extensión cofibración-fibración de A por B, a una sucesión exacta corta $0 \longrightarrow A-I \rightarrow E-D_{B} \rightarrow 0$, tal que $i$ es cofibración y p es fibración (respecto a la teoría de homotopia inducida por un anillo $\mathrm{R}$ en los grupos abelianos). Si B ( A) tiene una (co)presentación contraltil, damos un teorema de clasificación homotópica de las extensiones cofibración-fibra ción (salvo isomorfismo). Estudiamos las sucesiones exactas largas asociadas $\mathrm{y}$ analizamos el caso $\mathrm{R}=\emptyset$.

INTRODUCION.- En (4), para un anillo R, hernos definido en los grupos abelianos una teoría de homotopia, hemos estudiato propiedades de cofibraciones y fibraciones y construido sucesiones homotópicas; aquí utilizando los resultados de (4), es tudiamos el functor $\mathrm{CF}(\mathrm{B}, \mathrm{A})$. Asi como en (6), se da una clasificación homotópica de los G-fibrados principales y en (3) (5) se hace un estudio de $\operatorname{Ext}(B, A)$ a traves ae adecuadas presentaciones, nosotros hacemos una clasificación homotópica de las extensiones cofibración-fibración (salvo isomorfismo), que denotamos $C F(B, A)$, utilizando (co)presentaciones contractiles (cuando existen). Si el anillo que consideramos es Q, probainos que si $B$ es divisible, aamite una presentación contractil. $\Omega B \longrightarrow B \longrightarrow B$ y en este caso $C F(B, A)=[\Omega, A]$. Si A es de cotorsión y libre de torsión, tiene una copresentación contractil $A \longrightarrow A \longrightarrow G A$, entonces $C F(B, A)=[B, S A]$, además $\mathrm{CF}(\mathrm{B}, \mathrm{A})=\operatorname{Ext}(\mathrm{B}, \mathrm{A})$. 
A continuación, recogemos los teoremas más importantes, de jando al lector el enunciado de los correspondientes duales. con $A$ denotanos la categoría de los grupos abelianos.

EXTENSIONES COFIBRACION-FIBRACION. Vamos a considerar la categoría $\zeta F(B, A)$, que tiene como objetos las CF-extensiones de $A$ por $B$ (extensiones cofibración-fibreión), $u=(A \stackrel{i}{\longrightarrow} \stackrel{p}{\longrightarrow} B$ ), $u^{\prime}=\left(A \stackrel{i^{\prime}}{\longrightarrow} P^{\prime} \longrightarrow B\right)$ y como morfismos $f: u \longrightarrow \longrightarrow u^{\prime}$, homomorfismos $f: E \longrightarrow E^{\prime}$ tal que $f i=i^{\prime}, p^{\prime} f=p$. Es inmediato probar que todo morfismo de $\mathscr{G} F(B, A)$ es isomorfismo.

Para un homomorfismo $\alpha: A \longrightarrow K$, definimos un functor $\alpha_{\text {* }}: \zeta F(B, A) \longrightarrow \mathscr{F}(B, K)$, del modo siguiente, sea $f: u \longrightarrow u^{\prime}$ $y$ consideremos el siguiente diagrama:

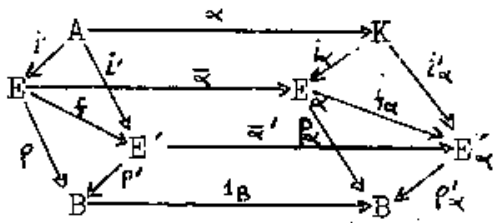

construido, a traves de los cuadrados cocartesianos determina dos por $\alpha$ e i primero, $y \alpha$ e $i^{\prime}$ despues, $f_{\alpha}=i_{\alpha}^{\prime} v \bar{\alpha}^{\prime} \cdot f$, entonces

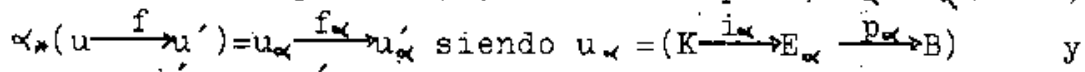
$u_{\alpha}^{\prime}=\left(K \stackrel{i_{\alpha}^{\prime}}{\rightarrow} \mathrm{E}_{\alpha}^{\prime} \stackrel{p_{\alpha}^{\prime}}{\longrightarrow} \mathrm{B}\right)$.

Definición.- Sean $u, v$ dos CF-extensiones de A por B, diremos que son equivalentes si existe un isomorfismo $f: u \longrightarrow \longrightarrow$. Denotaremos por CF(B,A) las Cr-extensiones de A por B, salvo isomorfismo.

Notemos que el functor $\alpha_{*} 10^{\circ}$ podemos considerar de modo na tural como una correspondencia $C \mathrm{~B}(\mathrm{~B}, \mathrm{~A}) \longrightarrow \mathrm{CF}(\mathrm{B}, \mathrm{K})$.

Lema.- Consideremos el siguiente diagrama:

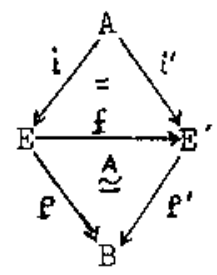


tal que p'es cofibreción, $p i=p^{\prime} i^{\prime}, f i=i^{\prime}, p \stackrel{A}{\simeq} p^{\prime} f$ entonces existe $f^{\prime}: E \longrightarrow E^{\prime}$ tal que $f^{\prime} \stackrel{A}{=} f \quad$ y $p^{\prime} f^{\prime}=p$.

Ayundndonos de este lema podemos probar la sirulente proposición:

Proposición.- Sean $\alpha^{\circ}, \alpha^{\prime}: A-K$ tal que $\alpha^{\prime} \simeq \alpha^{\prime}$-ntonces $\alpha_{n}^{0}=\alpha_{*}^{\prime}: \mathrm{CF}(B, A) \longrightarrow \mathrm{CB}(B, K)$.

Definición. - Sea $B \in|A|$ diremos que $\xi_{B}=\left(N_{B} \stackrel{j}{\longrightarrow} \mathrm{C} \stackrel{9}{\longrightarrow}\right.$ B) es ura presentación contractil de $B$, si $\zeta_{B}$ es iana Cr-extensión de A por $B$ y' $C$ es contractil.

Despues denostramos el siguiente teorema de clasificación Teorema. - Sea $B \in \mid$ Al y supongamos que $B$ tiene una presentación contractil $\xi_{B}=\left(N_{B} \stackrel{j}{\longrightarrow} \longrightarrow Q_{B}\right)$ entonces 1a correspondencia $\psi:\left[\mathrm{N}_{B}, A\right] \longrightarrow C F(B, A)$ tal que $\left.\psi[\alpha]=\alpha_{*}[]_{B}\right]$ es una equivalencia natural, en la segunda variable. La inversa $\varphi: \mathrm{CF}(\mathrm{B}, \mathrm{A}) \longrightarrow\left[\mathrm{N}_{\mathrm{B}}, \mathrm{A}\right]$ se define del siguiente modo $Y[A \stackrel{i}{-}-\mathrm{p}-\mathrm{B}]=[\hat{\mathrm{e}}]$ siendo $\mathrm{e}: \mathrm{C}-\mathrm{E}$ una elevación de $\mathrm{q}$ a traves de $p$, pe=q y ê el homomorfismo inducido en los conúcleos, único tal que $i \hat{e}=e j$.

Un corolario inmediato es la existencia de la siquientes sucesiones exactas.

Corolario.- Sea $B \in|A|$ y supongemos queB tiene una presentación contractil, sea un homomorfismo $g: X \longrightarrow \longrightarrow \mathrm{y}$ $\ldots \rightarrow \Omega Y \stackrel{i^{1}}{\longrightarrow} F \stackrel{g^{1}}{\rightarrow} X \stackrel{g}{\longrightarrow} Y$ la sucesión de Eckmann-Hilton asociada a $g$, entonces la siguiente sucesión es exacta para $n \geqslant l$. $\ldots \rightarrow \mathrm{CF}\left(\mathrm{B}, \Omega^{n} Y\right) \longrightarrow \mathrm{CF}\left(\mathrm{B}, \Omega^{n-1}{ }_{\mathrm{g}}\right) \longrightarrow \mathrm{CF}\left(\mathrm{B}, \Omega^{\mathrm{n}-1} \mathrm{X}\right) \rightarrow \mathrm{CF}\left(\mathrm{B}, \Omega^{\mathrm{n}-1} \mathrm{Y}\right)-$ Si además g es una fibración con núcleo $\mathrm{F} \stackrel{i}{\longrightarrow} \mathrm{X}$ y sucesión de fibras homotópicas asociada $\ldots \rightarrow \Omega Y \stackrel{\Delta}{\rightarrow} \stackrel{i}{\longrightarrow} \mathrm{X} \stackrel{\mathrm{g}}{\rightarrow} \mathrm{Y}$ la si $\cdots$ guiente sucesión es exacta para $n \geqslant l$.

$\ldots-C F\left(B, \Omega^{n} Y\right)-C F\left(B, \Omega^{n-1} F\right)-C F\left(B, \Omega^{n-1} X\right)-C F\left(B, \Omega^{n-1} Y\right)-$

Por último aseguramos que estas sucesiones son naturales respecto pares de homomorfismos como los siguientes: 


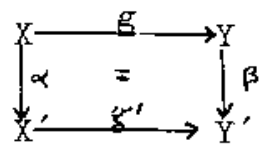

Ejemplos.- Para $R=Q$, si $B$ es divisible admite la siguiente

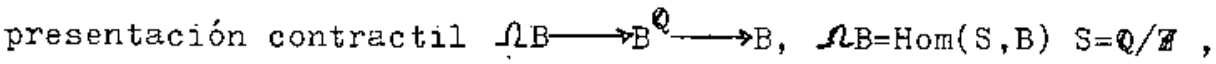
entonces $C F(B, A)=[\Omega B, A]$. Podenos ver que $C F \neq$ Ext ya que $\mathrm{CF}(Q, Z) \neq \operatorname{Ext}(Q, Z), \mathrm{CF}(Q, \mathbb{Z})=0$ por ser $Q$ contractil, $\mathrm{y}$ $\operatorname{Ext}(Q, \mathbf{Z}) \cong R$, Si $A$ es de cotorsión entonces $C F(B, A) \cong \operatorname{Ext}(B, A)$, si adenás $A$ es libre de torsión $A \longrightarrow A \otimes Q-A \otimes S$ es una copresentación contractil, entonces $\operatorname{Ext}(B, A) \simeq C F(B, A)=[B, A \otimes S]$ $\mathrm{Si}$ tomamos $\mathrm{B}=\mathrm{S}^{\mathrm{p}}=\mathbb{Z}\left(\mathrm{p}^{\infty}\right)$ y $\mathrm{A}=\mathrm{J}_{\mathrm{p}}$ enteros $\mathrm{p}$-adicos, entonces:

$$
\begin{aligned}
& \operatorname{Ext}\left(S^{p}, J_{p}\right) \cong \operatorname{CF}\left(S^{p}, J_{p}\right) \\
& \operatorname{CF}\left(S^{p}, J_{p}\right) \cong\left[\Omega S^{p}, J_{p}\right] \cong\left[J_{p}, J_{p}\right] \cong H_{0 m}\left(J_{p}, J_{p}\right) \cong J_{p} \\
& C P\left(S^{p}, J_{p}\right) \simeq\left[S^{p}, J_{p} \otimes S\right] \simeq \operatorname{Hom}\left(S^{p}, J_{p} \otimes S\right)
\end{aligned}
$$

\section{BIBLIOGRAFIA}

(1) Cartan,H; Eilemberg,S: Homological Algebra. Princeton University Press (1956).

(2) Dieck, T. tom; Kamps, K.H.; Puppe: Homotopietheorie. Lecture Notes in Mathematics. No 157. Springer-Verlag (1970) (3) Fuchs, L: Infinite Abelian Groups. Academic Press(1970) (4) Hernandez, L. J.: Un Ejemplo de Teoría de Homotopia en Grupos Abelianos (por apayecer)

(5) Hilton, P.J.I Stammbach,U.: A course in Homological Algebra, GTM 4, Springer-Verlag (1971). (6) Husemoller, D.:Fibre Bundies. Mc Graw-Hill(1966). 